\title{
BMJ Open Using a period incidence survey to compare antibiotic use in children between a university hospital and a district hospital in a country with low antimicrobial resistance: a prospective observational study
}

Christian Magnus Thaulow, ${ }^{1}$ Hege Salvesen Blix, ${ }^{2,3}$ Beate Horsberg Eriksen, ${ }^{1}$ Ingvild Ask, ${ }^{4}$ Tor Åge Myklebust, ${ }^{5}$ Dag Berild ${ }^{6}$

To cite: Thaulow CM, Blix HS, Eriksen $\mathrm{BH}$, et al. Using a period incidence survey to compare antibiotic use in children between a university hospital and a district hospital in a country with low antimicrobial resistance: a prospective observational study. BMJ Open 2018;9:e027836. doi:10.1136/ bmjopen-2018-027836

- Prepublication history and additional material for this paper are available online. To view, please visit the journal (http:// dx.doi.org/10.1136/bmjopen2018-027836).

Received 09 November 2018 Revised 18 February 2019 Accepted 03 May 2019
Check for updates

(C) Author(s) (or their employer(s)) 2019. Re-use permitted under CC BY-NC. No commercial re-use. See rights and permissions. Published by BMJ.

For numbered affiliations see end of article.

Correspondence to Dr Christian Magnus Thaulow; cmt85@hotmail.com

\section{ABSTRACT}

Objectives To describe and compare antibiotic use in relation to indications, doses, adherence rate to guidelines and rates of broad-spectrum antibiotics (BSA) in two different paediatric departments with different academic cultures, and identify areas with room for improvement.

Design Prospective observational survey of antibiotic use.

Setting Paediatric departments in a university hospital (UH) and a district hospital (DH) in Norway, 2017. The registration period was 1 year at the $\mathrm{DH}$ and 4 months at the UH.

Participants 201 children at the DH (mean age 3.8: SD 5.1) and 137 children at the UH (mean age 2.0: SD 5.9) were treated with systemic antibiotics by a paediatrician in the study period and included in the study.

Outcome measures Main outcome variables were prescriptions of antibiotics, treatments with antibiotics, rates of BSA, median doses and adherence rate to national guidelines.

Results In total, 744 prescriptions of antibiotics were given at the $\mathrm{UH}$ and 638 at the $\mathrm{DH}$. Total adherence rate to guidelines was $75 \%$ at the $\mathrm{UH}$ and $69 \%$ at the $\mathrm{DH}(\mathrm{p}=0.244)$. The rate of treatments involving BSA did not differ significantly between the hospitals $(p=0.263)$. Use of BSA was related to treatment of central nervous system (CNS) infections, patients with underlying medical conditions or targeted microbiological treatment in $92 \%$ and $86 \%$ of the treatments, at the $\mathrm{UH}$ and $\mathrm{DH}$, respectively $(p=0.217)$. A larger proportion of the children at the $\mathrm{DH}$ were treated for respiratory tract infections $(p<0.01)$ compared with the $\mathrm{UH}$. Children at the $\mathrm{UH}$ were treated with higher doses of ampicillin and cefotaxime $(p<0.05)$ compared with the $\mathrm{DH}$.

Conclusion Our results indicate that Norwegian paediatricians have a common understanding of main aspects in rational antibiotic use independently of working in a UH or DH. Variations in treatment of respiratory tract infections and in doses of antibiotics should be further studied.

\section{Strengths and limitations of this study}

- This paediatric study is based on individual patient data collected prospectively in a university hospital and a district hospital in a country with low antimicrobial resistance and includes information on antibiotic use, indications for treatment, underlying medical conditions, microbiological samples and doses.

No registration data were missing on the included children.

- The adherence rate to the national antibiotic guideline for common infections was calculated.

- Some case-mix differences in the two study populations made us carefully select outcomes that was comparable.

\section{INTRODUCTION}

Antimicrobial resistance (AMR) represents a serious threat to global health and is partly caused by inappropriate use of antibiotics. ${ }^{1-3}$ Exposure to antibiotics, especially broadspectrum antibiotics (BSA) in children, may also increase the risk of various chronic diseases. ${ }^{4-6}$ Use of BSA in Norwegian hospitals have increased during the last 10 years despite low resistance rates. ${ }^{78}$ Norway has a National Strategy against AMR including a $30 \%$ reduction in the use of $\mathrm{BSA}$ in hospitals within $2020^{9}$

Raastad et al revealed a significantly increased consumption of BSA in a highly specialised Norwegian paediatric department. ${ }^{10}$ Our group recently showed that a high number $(30 \%)$ of children in Norwegian general hospitals are receiving $\mathrm{BSA},{ }^{11}$ and that adherence rate to antibiotic guidelines is low $(48 \%)$. However, parameters such 
Table 1 Empirical recommendations for treatment of infections in Norwegian children

\begin{tabular}{ll}
\hline Indication & $\begin{array}{l}\text { First-line empirical recommendation in } \\
\text { the guideline* }\end{array}$ \\
\hline Pneumonia & $\begin{array}{l}\text { Phenoxymethylpenicillin or } \\
\text { benzylpenicillin }\end{array}$ \\
$\begin{array}{l}\text { Urinary tract } \\
\text { infection }\end{array}$ & $\begin{array}{l}\text { Aminoglycoside plus ampicillin } \\
\text { Pivmecillinam or amoxicillin/clavulanic } \\
\text { acid }\end{array}$ \\
$\begin{array}{l}\text { Sepsis and } \\
\text { neutropenia }\end{array}$ & $\begin{array}{l}\text { Aminoglycoside plus ampicillin } \\
\text { Infections in } \\
\text { skin, soft tissue, } \\
\text { bone and joint }\end{array}$ \\
$\begin{array}{l}\text { Cloxacillin, dicloxacillin, } \\
\text { clindamycin, cefalotin, cefalexin, } \\
\text { phenoxymethylpenicillin or } \\
\text { benzylpenicillin (alone or in } \\
\text { combination) }\end{array}$ \\
$\begin{array}{l}\text { Infection in ear, } \\
\text { eye and throat }\end{array}$ & $\begin{array}{l}\text { Phenoxymetylpenicillin or } \\
\text { benzylpenicillin (throat and ear) } \\
\text { Cefotaxime or clindamycin (severe } \\
\text { infections) }\end{array}$ \\
CNS infections & Cefotaxime or ceftriaxone
\end{tabular}

${ }^{*}$ First-line treatment options in the Norwegian guideline. ${ }^{28}$

CNS, central nervous system.

as underlying medical conditions, treatments based on microbiological samples and doses of antibiotics were not evaluated.

Differences in antibiotic prescription patterns for paediatric inpatients are observed between countries, ${ }^{12} 13$ and also within geographical areas. ${ }^{14-17} \mathrm{~A}$ low adherence rate to paediatric antibiotic guidelines is a global challenge both in hospitals and primary care, and especially in respiratory tract infections. ${ }^{18-20}$ Furthermore, there is no common international agreement regarding the optimal antibiotic dose for children in relation to body mass and type of infection. ${ }^{13}{ }^{21-24}$ In fact, scientific evidence does not give a clarifying answer on whether a higher or a lower dose of antibiotics will minimise the development of antibiotic resistance, ${ }^{25}$ but the mutant selection window theory indicate the importance of a high enough dose. ${ }^{26}$

In Norway, all acute care hospitals are public. There are 68 hospitals registered in the database of The Norwegian Institute of Public Health; 6 of these are university hospitals (UHs), while the remaining are smaller district hospitals (DHs). Twenty-three of the hospitals have a paediatric department. Comparing hospitals of different sizes and academic cultures gives a more valid description of antibiotic prescriptions throughout the country. The UHs are holding many academic positions and are expected to be role models in clinical practice for the DHs. We therefore speculate if there are any clearly differences in pattern of antibiotic use in children between centrally located UHs and more rural located DHs.

The primary aim of this study is to investigate whether use of BSA and adherence rate to antibiotic guidelines differs between children treated in a centrally located
$\mathrm{UH}$ and a more rural located DH. The secondary aim is to compare the distribution of indications for treatment, the duration of hospital treatment, route of administration, use of combination therapy, obtaining rate of blood cultures and doses of antibiotics. All aims are seen in the context of targeting areas for improvement of antibiotic use.

\section{METHODS}

\section{Study setting and design}

This is a prospective study using a period incidence design to compare paediatric antibiotic prescriptions in a $\mathrm{UH}$ (Oslo University Hospital, Ullevål) and a DH (Ålesund Hospital) in Norway, 2017. Neonatal and paediatric intensive care units were not included. In both hospitals, children $0-18$ years of age are admitted in paediatric departments.

\section{Hospitals}

Ålesund Hospital, hereafter called the DH, is located in the western part of Norway, and holds a wide range of medical specialty services. The paediatric ward consists of 18 beds. Data were collected during 12 months in 2017, from 1 January to 31 December.

The paediatric department in Oslo University Hospital, Ullevål (UH) consists of various wards. We collected data from the paediatric infectious ward (18 beds) during 4 months in 2017, from 1 June to 31 July and from 17 October to 17 December. In the period from 1 to 31 July, the general paediatric ward was merged with the paediatric infectious and observation ward because of summer holiday and included in our registration.

The UH is a national referral centre for children with cystic fibrosis, but does on the other hand not admit oncological or cardiological patients. The DH does not have any national services, but treat children with all kinds of clinical conditions. In opposite to the DH, the UH has many paediatricians holding academic positions working in close collaboration with the clinicians.

\section{Data collection}

The data were collected from the medical records every day at 08:00 in both hospitals. In the DH, this was done by trained nurses working on the ward and double-checked by a medical doctor every day. In the UH, one paediatrician did all registrations, and the quality control was performed by the head of this project. For registrations, we applied an international standardised point prevalence protocol developed by the European Centre for Disease Prevention and Control (ECDC),${ }^{27}$ and the data were stored in an electronical database (without national identification numbers). Educational classes to doctors and nurses who were data collectors were held before the start of registration in both hospitals.

Data collection included the total number of patients in the wards, national identification numbers, gender, age, weight, underlying medical conditions, type and 
Table 2 Antibiotic prescriptions by paediatricians for treatment of infections in a Norwegian university hospital and a district hospital (only in-hospital prescriptions)

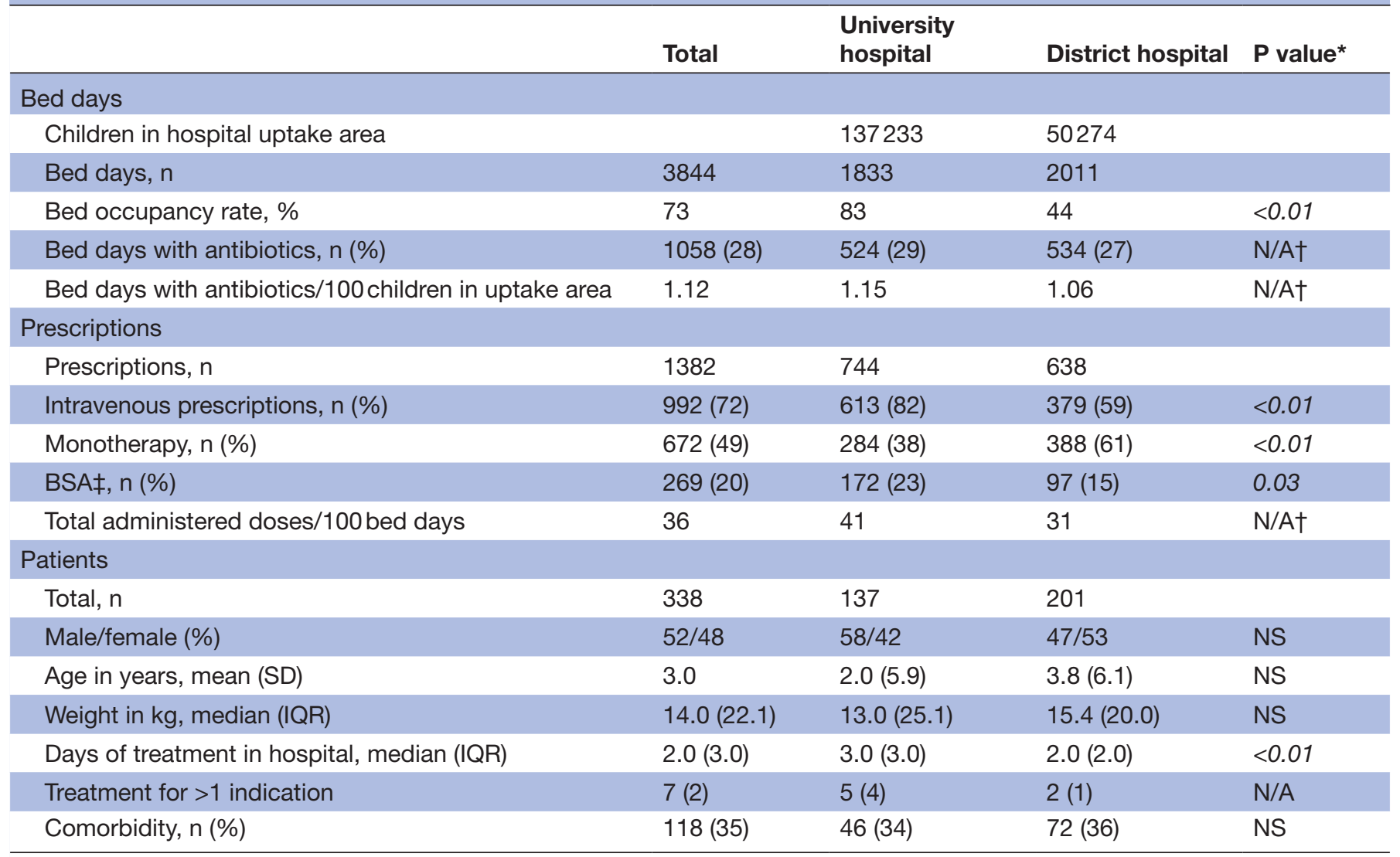

${ }^{*} \mathrm{~A} \chi^{2}$ test was used for proportions, Student's t-test for means and Moods median test for medians.

†In the district hospital, all paediatric bed days were included, but in the university hospital only those admitted to the infectious ward were included. A statistical comparison of total antibiotic use was therefore not performed.

‡Broad-spectrum antibiotics (BSA): second-generation and third-generation cephalosporins, carbapenems, piperacillin/ tazobactam, carbapenems and ceftolazan/tazobactam. P-value $<0.05$ was regarded significant.

dose of antibiotics, route of administration, whether it was for treatment or prophylaxis, indication for antibiotic treatment and whether the infection was healthcare or community acquired. Results from blood cultures, bone/ joint aspirations and airway samples from patients with cystic fibrosis were registered.

\section{Definitions}

Definitions of underlying medical conditions and the clinical indication for treatment derived from the ECDC rules for conducting a point-prevalence survey ${ }^{27}$ and were reported based on predefined lists. Less severe medical conditions such as allergies and asthma without daily medication were not registered as comorbidities. Surgical prophylaxis was defined as antibiotics given immediately before, during or shortly after surgery to prevent infection. Medical prophylaxis was defined as antibiotics prescribed to prevent infection in patients at risk. Healthcare-associated infections were defined according to the ECDC criteria. ${ }^{27}$ Antibiotics were defined as antibacterials for systemic use (J01), oral vancomycin (A07AA09) and oral metronidazole (P01AB01). Tuberculostatics (eg, rifampicin) were not included. BSA were defined as second-generation and third-generation cephalosporins, ceftolozane/tazobactam, carbapenems, piperacillin/ tazobactam and quinolones, according to the National Strategy against AMR. ${ }^{9}$

\section{Guidelines}

To evaluate adherence to guidelines, we used empirical recommendations given in Norwegian Guidelines-Acute Paediatrics by The Norwegian Pediatric Association. ${ }^{28}$ Treatments in accordance with susceptibility patterns from blood cultures, bone/joint aspirations and airway samples in patients with cystic fibrosis were also included when calculating the adherence rate. A summary of the empirical recommendations is shown in table 1.

An adherence rate of at least $65 \%$ was regarded as satisfactory based on our previous study. ${ }^{11}$ When evaluating doses of antibiotics, we used the British National Formulary for Children, ${ }^{29}$ because it is commonly used by Norwegian paediatricians. We did not evaluate whether treatment with antibiotics was indicated in the first place, nor the length of the treatment, only choices of antibiotics and doses. 
Distribution of antibiotic doses
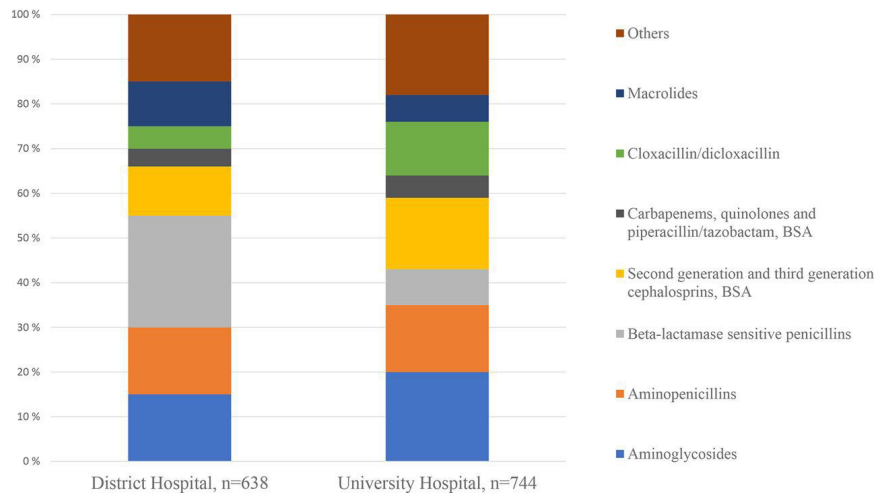

Figure 1 Distribution of antibiotic doses given by paediatricians for treatment of infection to hospitalised children in a Norwegian district hospital and a university hospital. Broad-spectrum antibiotics (BSA) and all other antibiotics accounting for $>10 \%$ of total doses in one of the hospitals were included.

\section{Analyses}

To minimise case-mix variations between the hospitals, only admissions of patients treated by paediatricians (not surgeons) and antibiotics issued for treatment of infections (not prophylaxis) were included in our analyses. Antibiotic use was described in relation to bed days, total number of antibiotic prescriptions, proportion of admitted patients receiving antibiotics and the total number of antibiotic treatments. One prescription was defined as a daily dose with one antibiotic, and treatment was defined as antibiotic therapy for a certain indication in a certain time range. Doses were described and compared in $\mathrm{mg} / \mathrm{kg} /$ day only for children $<40 \mathrm{~kg}$, and we controlled for hospital differences in distribution of indications and weight by doing stratified analyses. When comparing adherence rate to guideline and BSA use we adjusted for age. We also controlled for the impact of seasonal variation by analysing data on treatments with identical registration periods.

\section{Statistics}

Statistical analyses were performed using Microsoft excel 2016 and SPSS Statistics V.23. The proportion of children receiving antibiotics was described separately for each hospital without statistically comparisons because of case mix. For all other analyses including BSA rates $(\%)$, comorbidity rates $(\%)$, age (mean), duration of treatment (median), doses (median) and route of administration $(\%)$, comparisons were done using either $\chi^{2}$ test (proportions), Student's t-test (means) or Moods median test (medians). Fishers exact test was used to analyse differences in distribution of indications when comparing doses. When comparing adherence to guidelines and use of BSA, we controlled for age differences between the hospitals by using a multivariable logistic regression analyse adjusting for age as an independent variable. A $\mathrm{p}<0.05$ was considered significant. SD was used in relation to means and IQR in relation to medians. No data were missing for the statistical analyses.

\section{Patient and public involvement}

This study is part of a comprehensive project, 'Born in the sunset of antibiotics - use of antibiotics in hospitalized children in a country with low antimicrobial resistance'. For this project, we have recently recruited a user representative from The Norwegian Society of Children's Cancer. She has received the project protocol, but not been directly involved in the conduction of this specific study; however, she will help implement our results to the general population and take more actively part in upcoming studies.

\section{RESULTS}

\section{General demographics}

In total, 3844 bed days (1833 at the UH and 2011 at the $\mathrm{DH})$ were registered, whereof $28 \%$ (29\% at the UH and $27 \%$ at the $\mathrm{DH}$ ) included exposure to antibiotic therapy (table 2).

The proportion of intravenous infusions and combination of antibiotics were significantly higher at the UH compared with the DH $(p<0.01)$. Thirty-four per cent of patients at the $\mathrm{UH}$ and $36 \%$ at the $\mathrm{DH}$ had an underlying medical condition. No fatalities were registered during the study periods.

\section{Total antibiotic use}

Beta-lactamase susceptible penicillins accounted for the highest proportion $(25 \%)$ of antibiotic prescriptions at the DH compared with $8 \%$ at the UH $(\mathrm{p}<0.01)$. Aminoglycosides represented the highest proportion (20\%) at the UH compared with $15 \%$ at the $\mathrm{DH}$ (figure 1).

\section{Indications for treatment with antibiotics and adherence rate to the guideline}

Of all 345 treatments, $32 \%$ where given for pneumonia (table 3 ). At the $\mathrm{DH}$, a higher proportion of treatments were given for pneumonia $(p<0.01)$ and upper respiratory tract infections $(\mathrm{p}<0.01)$ compared with the $\mathrm{UH}$, while more patients at the UH were treated for infections in skin, soft tissue, bone and joint $(p<0.01)$. For infections in skin, soft tissue, bone and joint, 9 (27\%) out of 33 treatments at the UH and $10(43 \%)$ out of 33 treatments in the DH involved clindamycin $(\mathrm{p}=0.176)$. Total adherence to guideline was $72 \%$, varying for different indications, and without significant differences between hospitals (table 3). Treatments for pneumonia had the lowest adherence rate to the guideline; $25 \%$ of the treatments involved erythromycin at the $\mathrm{UH}$ and $18 \%$ at the $\mathrm{DH} ; 13 \%$ of the treatments involved aminopenicillins or trimethoprim-sulfamethoxazole at the $\mathrm{UH}$ and $15 \%$ at the DH.

\section{Use of BSA}

The proportion of treatments involving BSA varied for different indications, but we revealed no significant 
Table 3 Paediatric antibiotic prescriptions for different indications in a Norwegian university hospital and a district hospital

\begin{tabular}{|c|c|c|c|c|}
\hline Indications for antibiotic treatment & Total & $\begin{array}{l}\text { University } \\
\text { hospital }\end{array}$ & $\begin{array}{l}\text { District } \\
\text { hospital }\end{array}$ & P value* \\
\hline \multicolumn{5}{|l|}{ All indications $\dagger$} \\
\hline Treatments, $\mathrm{n}$ & 345 & 142 & 203 & \\
\hline Healthcare-acquired infections, $\mathrm{n}(\%)$ & $17(5)$ & $11(8)$ & $6(3)$ & 0.04 \\
\hline Treatments involving BSA $\ddagger$, n (\%) & $72(21)$ & $34(24)$ & $38(19)$ & NS \\
\hline Treatments according to guideline§ף, $n$ (\%) & $232(72)$ & $96(75)$ & $136(69)$ & NS \\
\hline \multicolumn{5}{|l|}{ Pneumonia } \\
\hline Treatments, $\mathrm{n}$ (\% of all treatments) & $110(32)$ & $32(23)$ & $78(38)$ & $<0.01$ \\
\hline Treatments involving BSA, n (\%) & $24(22)$ & $11(34)$ & $13(17)$ & NS \\
\hline Treatments according to guideline, $n(\%)$ & $57(52)$ & $15(47)$ & $42(54)$ & NS \\
\hline Days of treatment in hospital, median (IQR) & $2.0(3.0)$ & $3.0(4.5)$ & $2.0(2.3)$ & NS \\
\hline Treatments to patients with comorbidities, $\mathrm{n}(\%)$ & $54(49)$ & $19(59)$ & $35(45)$ & NS \\
\hline BSA treatments to patients with comorbidities ${ }^{* *}, n$ (\% of BSA) & $23(96)$ & $11(100)$ & $12(92)$ & NS \\
\hline \multicolumn{5}{|l|}{ Urinary tract infection } \\
\hline Treatments, $\mathrm{n}$ (\% of all treatments) & $59(17)$ & $28(20)$ & $31(15)$ & NS \\
\hline Treatments involving BSA, $n(\%)$ & $6(2)$ & $2(7)$ & $4(13)$ & NS \\
\hline Treatment according to guideline, $\mathrm{n}(\%)$ & $50(85)$ & $26(93)$ & $24(77)$ & NS \\
\hline Days of treatment in hospital, median (IQR) & $2.0(2.0)$ & $2.0(1.8)$ & $3.0(3.0)$ & NS \\
\hline Treatments to patients with comorbidities, $\mathrm{n}(\%)$ & $20(34)$ & $7(25)$ & $13(42)$ & NS \\
\hline BSA treatments to patients with comorbidities, $n$ (\% of BSA) & $5(83)$ & $2(100)$ & $3(75)$ & N/A \\
\hline \multicolumn{5}{|l|}{ Infection in skin, soft tissue, bone and joint } \\
\hline Treatments, $\mathrm{n}$ (\% of all treatments) & $56(16)$ & $33(23)$ & $23(11)$ & $<0.01$ \\
\hline Treatments involving BSA, $\mathrm{n}(\%)$ & $8(14)$ & $6(18)$ & $2(9)$ & NS \\
\hline Treatments according to guideline, $n(\%)$ & $41(73)$ & $23(70)$ & $18(78)$ & NS \\
\hline Days of treatment in hospital, median (IQR) & $2.0(4.0)$ & $3.0(5.0)$ & $1.5(3.0)$ & NS \\
\hline Treatments to patients with comorbidities, $\mathrm{n}(\%)$ & $10(18)$ & $1(3)$ & $9(38)$ & $<0.01$ \\
\hline BSA treatments to patients with comorbidities, $n$ (\% of BSA) & $1(10)$ & $0(0)$ & $1(50)$ & N/A \\
\hline \multicolumn{5}{|l|}{ Sepsis } \\
\hline Treatments, $\mathrm{n}$ (\% of all treatments) & $34(9)$ & $19(13)$ & $15(7)$ & NS \\
\hline Treatments involving BSA, $n(\%)$ & $7(21)$ & $4(21)$ & $3(20)$ & NS \\
\hline Treatments according to guideline, $\mathrm{n}(\%)$ & $30(88)$ & $18(95)$ & $12(80)$ & NS \\
\hline Days of treatment in hospital, median (IQR) & $3.5(4.0)$ & $3.0(3.0)$ & $5.0(5.0)$ & NS \\
\hline Treatments to patients with comorbidities, $\mathrm{n}(\%)$ & $14(41)$ & $8(42)$ & $6(40)$ & NS \\
\hline $\mathrm{BSA}$ treatments to patients with comorbidities, $\mathrm{n}$ (\% of BSA) & $6(86)$ & $3(100)$ & $3(75)$ & N/A \\
\hline \multicolumn{5}{|l|}{ Upper respiratory tract infections } \\
\hline Treatments, $\mathrm{n}(\%)$ & $42(12)$ & $7(5)$ & $35(17)$ & $<0.01$ \\
\hline Treatments involving BSA, $n(\%)$ & $2(5)$ & $1(14)$ & $1(3)$ & NS \\
\hline Treatments according to guideline, $n(\%)$ & $34(81)$ & $7(100)$ & $27(77)$ & NS \\
\hline Days of treatment in hospital, median (IQR) & $2.0(2.0)$ & $2.0(2.0)$ & $2.0(2.0)$ & NS \\
\hline Treatments to patients with comorbidities, $\mathrm{n}(\%)$ & $10(24)$ & $2(29)$ & $8(23)$ & NS \\
\hline BSA treatments to patients with comorbidities, $n$ (\% of BSA) & $1(50)$ & $1(100)$ & $0(0)$ & $\mathrm{N} / \mathrm{A}$ \\
\hline \multicolumn{5}{|l|}{ CNS infections } \\
\hline Treatments, n (\%) & $23(7)$ & $9(6)$ & $14(7)$ & NS \\
\hline Treatment involving BSA, $\mathrm{n}(\%)$ & $20(87)$ & $7(78)$ & $13(93)$ & NS \\
\hline Treatments according to guideline, $n(\%)$ & $20(87)$ & $7(78)$ & $13(93)$ & NS \\
\hline
\end{tabular}


Table 3 Continued

\begin{tabular}{|c|c|c|c|c|}
\hline Indications for antibiotic treatment & Total & $\begin{array}{l}\text { University } \\
\text { hospital }\end{array}$ & $\begin{array}{l}\text { District } \\
\text { hospital }\end{array}$ & P value* $^{*}$ \\
\hline Days of treatment in hospital, median (IQR) & $1.0(2.0)$ & $2(3.0)$ & $1(1.25)$ & NS \\
\hline BSA treatments to patients with comorbidities, $n$ ( $\%$ of BSA) & $1(5)$ & $1(14)$ & $0(0)$ & NS \\
\hline \multicolumn{5}{|l|}{ Other infections } \\
\hline Treatments with BSA, n (\%) & $5(24)$ & $3(21)$ & $2(29)$ & NS \\
\hline
\end{tabular}

${ }^{*} \mathrm{~A} \chi^{2}$ test was used for proportions and Moods median test for medians. Non-significant results are marked NS. N/A means that the numbers are too small for statistical testing.

†For adherence rate and BSA use, we controlled for age differences between the hospitals by using multivariable logistic regression, and the significant levels remained the same for all indications. P-value $<0.05$ was regarded significant.

‡Broad-spectrum antibiotics (BSA) were defined as second-generation and third-generation cephalosporins, carbapenems, piperacillin/ tazobactam and ceftolazan/tazobactam.

$\S$ The entire treatment is in adherence with the empirical recommendation in The Norwegian guideline ${ }^{28}$ and/or in accordance with blood cultures, bone/joint cultures or respiratory tract samples from cystic fibrosis patients (means using any antibiotic(s) that was susceptible by the bacteria, regardless of how the patient was treated initially).

ๆOther infections were not included when calculating total compliance with guidelines.

**University hospital: cystic fibrosis with pathogenic bacteria (9), cerebral palsy (1), recent CNS operation (1), district hospital: cystic fibrosis with pathogenic bacteria (8), lymphoma (1), neurological multifunction disability (1), heart disease (1), syndrome (1).

CNS, central nervous system.

differences between the hospitals (table 3). For urinary tract infections and pneumonia, nearly all treatments involving BSA (28 out of 30) were given to patients with an underlying medical condition. In cases of pneumonia, cystic fibrosis accounted for $17(71 \%)$ of treatments to patients with comorbidities. Prescriptions to patients with an underlying (mostly severe) medical condition, central nervous system (CNS) infection or treatment based on a microbiological sample, accounted for $90 \%$ of all doses with BSA (table 4). In the UH, nine admissions of patients with cystic fibrosis accounted for 91 (53\%) of total prescriptions with BSA while in the $\mathrm{DH}$, eight admissions of patients with cystic fibrosis were given 19 (20\%) of total prescriptions with BSA $(\mathrm{p}<0.01)$. When excluding patients with cystic fibrosis, no significant difference in prescription rate of BSA was found between the hospitals.

\section{Blood cultures}

In the UH, blood cultures were obtained before or during $77 \%$ of all treatments, as opposed to $44 \%$ in the DH $(p<0.01)$ (see online supplemental digital content 1 , table showing rates for various indications). Out of 14 positive blood cultures, Staphylococcus aureus was the most common bacteria (two cases in both hospitals). One case of extended spectrum beta-lactamase (ESBLE) was registered at the UH (see online supplemental digital content 2 for the results and treatments of all infections with positive blood cultures).

\section{Antibiotic doses}

Overall, the median dose in $\mathrm{mg} / \mathrm{kg} /$ day given to children $<40 \mathrm{~kg}$ was higher in the UH for six out of the seven most commonly prescribed antibiotics given intravenously. A significant difference was found for ampicillin and cefotaxime (figure 2). For ampicillin, we subgrouped the children above and below $10 \mathrm{~kg}$, and the difference was only significant for children $<10 \mathrm{~kg}(\mathrm{p}<0.01)$ with a median dose of $151 \mathrm{mg} / \mathrm{kg} /$ day in the $\mathrm{DH}$ and $199 \mathrm{mg} / \mathrm{kg} /$ day in the UH. For neonatal infants ( $<28$ days), ampicillin was administered three times a day to nearly all patients in both hospitals (three out of three in the DH, and six out of eight in the UH). For all remaining children $<40 \mathrm{~kg}$, ampicillin was mainly administered four times a day in the UH $(93 \%)$, and three times a day in the DH $(54 \%)(\mathrm{p}<0.01)$. Cefotaxime was also mainly administered four times a day in the UH ( 8 out of $11,73 \%$ ) and three times a day in the DH $(8$ out of $11,73 \%)(\mathrm{p}<0.01)$. The IQR was smaller in the UH for eight of the nine antibiotics. When comparing doses, we controlled for different indications for antibiotic therapy between the hospitals, and only found significant difference in the distribution of indications for ceftriaxone ( $\mathrm{p}=0.02)$ (see online supplemental digital content 3 for a detailed description of dose comparison).

\section{Seasonal variation}

To control for seasonal variation bias in the distribution of indications and choice of antibiotics, we analysed data from the DH corresponding directly with the collection periods at the UH (see online supplemental digital content 4). We revealed no significant differences in adherence rate or BSA use between the hospitals, and the differences in proportions of treatments being pneumonia or upper respiratory tract infections were significant at the same levels as in our main analyses. Also, the number of treatments at the $\mathrm{DH}$ was on the same level in the two periods; 65 during the 4 months that we collected data at the UH and 203 during all 12 months. 
Table 4 Overview of paediatric prescriptions with broad-spectrum antibiotics (BSA) in a Norwegian university hospital compared with a district hospital

\begin{tabular}{|c|c|c|c|c|}
\hline Prescriptions with BSA & Total & $\begin{array}{l}\text { University } \\
\text { hospital }\end{array}$ & $\begin{array}{l}\text { District } \\
\text { hospital }\end{array}$ & P value* \\
\hline All $B S A, \mathrm{n}$ & 269 & 172 & 97 & \\
\hline Prescriptions to patients with comorbidities, n (\%) & $182(68)$ & $120(70) \dagger$ & $62(64) \ddagger$ & NS \\
\hline Prescriptions to patients with cystic fibrosis, n (\%) & $110(41)$ & $91(53)$ & $19(20)$ & $<0.01$ \\
\hline Prescriptions to patients with CNS infections, $\mathrm{n}(\%)$ & $40(15)$ & $19(11)$ & $21(22)$ & 0.03 \\
\hline Prescriptions based on microbiological samples, $\mathrm{n}(\%)$ & $130(48)$ & $111(65) \S$ & $19(20) \emptyset$ & $<0.01$ \\
\hline $\begin{array}{l}\text { Prescriptions to patients with comorbidities, CNS infections or } \\
\text { based on microbiological samples, } \mathrm{n}(\%)\end{array}$ & $241(90)$ & $158(92)$ & $83(86)$ & NS \\
\hline $\begin{array}{l}\text { Second-generation and third-generation cephalosporines, } n \text { (\% of } \\
\text { BSA) }\end{array}$ & $186(69)$ & $116(67)$ & $70(72)$ & NS \\
\hline Prescriptions to patients with comorbidities, n (\%) & $103(55)$ & $65(56)$ & $38(54)$ & NS \\
\hline Prescriptions to patients with CNS infections, $\mathrm{n}(\%)$ & $40(22)$ & $19(16)$ & $21(30)$ & 0.02 \\
\hline Prescriptions based on microbiological samples, n (\%) & $76(41)$ & $57(49)$ & $19(27)$ & 0.03 \\
\hline Carbapenems, $n$ (\% of BSA) & $34(13)$ & $24(14)$ & $10(10)$ & NS \\
\hline Prescriptions to patients with comorbidities, n (\%) & $31(91)$ & $24(100)$ & $7(70)$ & NS \\
\hline Prescriptions based on microbiological samples, n (\%) & $24(77)$ & $24(100)$ & $0(0)$ & $<0.01$ \\
\hline Piperacillin-tazobactam, $\mathrm{n}$ (\% of BSA) & $28(10)$ & $17(10)$ & $11(11)$ & NS \\
\hline Prescriptions to patients with comorbidities, n (\%) & $28(100)$ & $17(100)$ & $11(100)$ & NS \\
\hline Prescriptions based on microbiological samples, n (\%) & $0(0)$ & $16(94)$ & $0(0)$ & $<0.01$ \\
\hline Quinolones, $\mathrm{n}$ (\% of BSA) & $7(3)$ & $1(0.6)$ & $6(6)$ & 0.01 \\
\hline Prescriptions to patients with comorbidities, n (\%) & $7(100)$ & $1(100)$ & $6(100)$ & $\mathrm{N} / \mathrm{A}$ \\
\hline Prescriptions based on microbiological samples, n (\%) & $1(14)$ & $1(100)$ & $0(0)$ & $\mathrm{N} / \mathrm{A}$ \\
\hline Ceftolozane/tazobactam, n (\% of BSA) & $14(5)$ & $14(8)$ & $0(0)$ & 0.02 \\
\hline Prescriptions to patients with comorbidities, $\mathrm{n}(\%)$ & $14(100)$ & $14(100)$ & $0(0)$ & $\mathrm{N} / \mathrm{A}$ \\
\hline Doses based on microbiological samples, $\mathrm{n}(\%)$ & $14(100)$ & $14(100)$ & $0(0)$ & N/A \\
\hline
\end{tabular}

${ }^{*} \chi^{2}$ test. Non-significant results are marked NS. N/A means that the numbers are too small for statistical comparisons.

†Cystic fibrosis (91), chronical kidney disease (9), neurological disease (6), others (14).

¥Cystic fibrosis (19), malignancy (14), inflammatory bowel disease (14), chronical kidney disease (9), neurological disease (5), heart disease (1).

§Blood cultures: extended spectrum beta-lactamase (ESBL) (3), Klebsiella pneumoniae (5), bone aspiration: Kingella kingae (12), airway sample: Pseudomonas aeruginosa (81), Mycobacterium abscessus (10).

ๆAirway sample: Pseudomonas aeruginosa (19).

There was no unusual outbreak of any microorganism during the study periods, but we do not have data about seasonal epidemics of common viruses like influenzae and respiratory syncytial virus (RSV).

\section{DISCUSSION}

\section{Principal findings}

This study reveals that both hospitals mostly prescribe BSA to patients with severe underlying medical conditions, for CNS infections and/or based on microbiological samples. Adherence to the guideline was high for most indications without significant differences between the hospitals. This indicate that Norwegian paediatricians have a common understanding of main aspects in rational antibiotic use independently of working in a $\mathrm{UH}$ or $\mathrm{DH}$.

\section{Limitations and strengths of the study}

There are some case-mix differences between the hospitals. Optimally, the general paediatric ward at the UH should also have been included in the comparison as it may have impacted some of our comparisons and inhibited us in performing statistical comparisons of total prescription rates. We have tried to minimise this problem by excluding antibiotics issued as prophylaxis. Information about antibiotic sales to the general ward was also analysed (data not shown), and we conclude that the exclusion of this ward would not have affected our main findings. Furthermore, oncological and cardiological patients are not admitted to the UH, but to another hospital in Oslo. However, only six of the patients who received antibiotics at the $\mathrm{DH}$ had a malignancy. Other factors related to different settings, such as bed occupancy 
Comparison of antibiotic dosages between a Norwegian university hospital and a district hospital

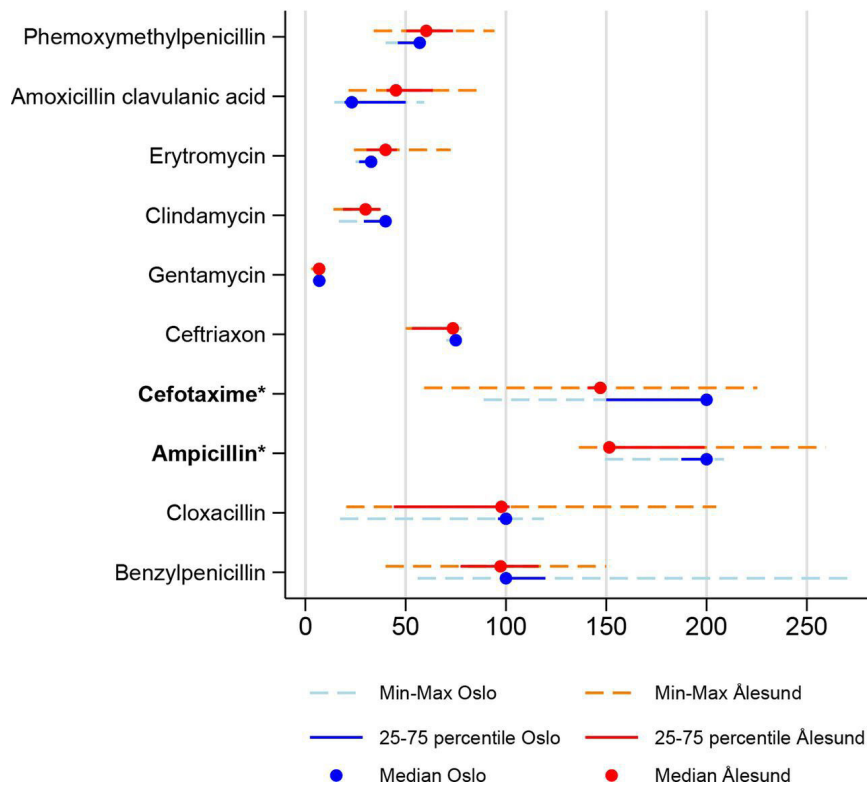

Figure 2 Comparison of Prescribed Daily Dose (PDD) of common antibiotics in children $<40 \mathrm{~kg}$ in a Norwegian university hospital and a district hospital. ${ }^{*} \mathrm{P}$-value $<0.05$, Moods median test.

rate, different composition of population in uptake area and the difference in geographical catchment area between the hospitals, may also have influenced our analyses. When calculating the adherence rate to the guideline, we did not evaluate whether antibiotic therapy was appropriate in first place, the severity of infection or the total duration of treatment; this is, important to be aware of when interpreting our data and are important quality indicators in antibiotic stewardship that should be evaluated in future studies.

Unfortunately, we were not able to organise one entire year of data collection from the UH. Different registration periods could have affected the incidence of infections, prescription rate and the choice of antibiotics, especially for respiratory tract infections. Online supplemental digital content 4 shows that our main conclusions are most certainly not affected by the different registration periods. Minor differences in the numbers for some of the non-respiratory tract infections are more likely to be caused by very small numbers.

A strength of this study is that a long-term period incidence registration limits the chance of temporary casualties like a seasonal epidemic. The inclusion of variables such as comorbidities, microbiological samples and doses further strengthen the results. By conducting the datacollection ourselves, we achieved to collect all necessary data on every single patient and we could process the data based on first-hand knowledge of the registration. This is in opposite to a previous Norwegian point prevalence study were data included less details and were based on a national registry. ${ }^{11}$
Are there clinically relevant differences in antibiotic use between the hospitals?

Children admitted to the UH were prescribed significantly more BSA than in the DH, but this was explained by long-term treatments of patients with cystic fibrosis in the UH. The difference between the hospitals was not significant when comparing BSA use based on the proportion of treatments involving BSA. Our primary aim was to investigate whether use of BSA and adherence rate to antibiotic guidelines differed between the hospitals; we found no reasons to believe that, indicating that the challenges related to these important quality markers in antibiotic stewardship are evaluated with a unified agreement among paediatricians in Norway regardless of working in a UH or DH. A previous study did neither find significant differences in use of BSA between a number of Norwegian anonymous hospitals and increases the generalisability of our results. ${ }^{11}$ Nevertheless, more patient-level analyses from other parts of Norway are needed to draw conclusions on prescription practice in other hospitals.

Evaluating our secondary aim, our results revealed several differences between the hospitals. The extensive use of intravenous infusions and combination therapy at the UH can partly be explained by the high number of children with cystic fibrosis receiving long-term combination therapy at the UH. The high use of oral antibiotics at the DH may indicate less severe infections but can also be explained by an early switch from intravenous to oral administration. A systematic review suggests that intravenous to oral switch can occur earlier than previously recommended for many indications. ${ }^{30}$

The distribution of indications varied between the hospitals, mostly due to the significant difference in number of treatments for pneumonia and upper respiratory tract infections. One hypothesis could be that these patients more often were treated as outpatients at the UH. This is supported by a much higher bed occupancy rate and a smaller geographical catchment area for the UH. Finally, the paediatricians at the UH may have regarded more respiratory tract infections as viral. To differ between viral and bacterial aetiology in pneumonia is a main challenge among paediatricians and studies show that most infections are viral. ${ }^{31} 32$ The UH have a closer collaboration with the microbiological department and an easier access to an extended panel of swabs and PCR, but analyses for commonly pathogens like Mycoplasma pneumoniae, RSV, rhinovirus and influenza viruses are easy and rapidly available in both hospitals.

One could hypothesise that patients treated at the UH in general were more severely ill, reflected by the higher proportion of blood cultures taken. However, this may also be explained by different traditions in the practice and involvement from the microbiological department.

Doses were generally higher at the UH compared with the DH. A European study showed wide variations in antibiotic dosing between different hospitals. ${ }^{13}$ According to the guideline, ${ }^{29}$ ampicillin and cefotaxime should be given four times a day for children after the neonatal 
period, and this represents an area of improvement for the DH. Wider IQR at the DH may indicate that doses were evaluated on a more individual basis rather than standardised. There is a need for studies on antimicrobial dosing in children as recommendations vary between guidelines. ${ }^{29} 33$

\section{Interpretation of the results in relation to the guideline and other studies}

Compared with the national point prevalence surveys, ${ }^{11}$ use of BSA seems lower in both of our study hospitals, especially when taking into account that prophylaxis was not included. The inclusion of bacterial samples in our study probably explain the high adherence rate to guideline compared with the national survey. ${ }^{11}$ Also, compared with international surveys, the children in our study received less BSA and more aminoglycosides. ${ }^{12} 1316$

For pneumonia, only half of the treatments were in adherence to the guideline in both hospitals, explained by a high use of other narrow-spectrum antibiotics than beta-lactamase susceptible penicillins, especially erytromycin, aminopenicillins and trimethoprim sulfamethoxazole. As vaccines for pneumococcus has been offered to all Norwegian children since 2006, clinicians may think that more pneumonias are caused by other bacteria than pneumococcus. However, a study from 2016 showed that pneumococcus remained the single bacteria accounting for most cases of paediatric pneumonia in Norway. ${ }^{31}$ Erytromycin may have been used in cases with PCR positive Mycoplasma samples from the nasopharynx. A Cochrane report did, however, not show any clinical benefits of empirical routine coverage for atypical bacteria in pneumonia in adults. ${ }^{34}$

For infections in skin, soft tissue, bone and joint, we were surprised to find extensive use of clindamycin. Even though clindamycin is recommended for severe infections, cloxacillin/dicloxacillin is preferable from an ecological point of view. ${ }^{35}$ The short median duration of hospital treatment argues against a high proportion of clinically severe infections. The poor availability of an oral mixture for cloxacillin/dicloxacillin in Norway (not registered by Norwegian authorities) may partly explain the high use of clindamycin which is easily accessible in mixture form. We do not know how many of our patients having penicillin allergy, but both erythromycin and clindamycin are in these cases recommended treatment for their respective indications. However, the prevalence of true penicillin allergy is estimated to be very low, only $0.01 \%-0.05 \%,{ }^{36}$ and one study found that among children who reported to have penicillin allergy, only around $20 \%$ had true allergy. ${ }^{37}$

\section{Practical implications of the study}

The study illuminates the antibiotic consumption in paediatric inpatients in a high-income country with a uniform and stable public healthcare system. The results can be applied in further antibiotic stewardship both in Norway and comparable countries. In Norway, the results should be evaluated against recommendations in the existing antibiotic guideline in a broader context than just calculating an adherence rate. The study will hopefully also inspire other hospitals to publish individual patient data on antibiotic consumption. Finally, future studies from hospitals should target other important quality indicators such as duration of treatment and whether antibiotics are indicated in first place.

\section{CONCLUSION}

Based on this study, we found no reasons to believe that use of BSA and adherence rate to antibiotic guidelines vary significantly between Norwegian $\mathrm{UH}$ and $\mathrm{DH}$.

We revealed that $\sim 3 / 4$ of the antibiotic treatments were in adherence with the guideline and that use of BSA mostly were related to severe underlying medical conditions, CNS infections and/or microbiological samples. Several issues need further investigation; the large proportional difference between the hospitals in children treated for respiratory tract infections; the high use of other antibiotics than beta-lactam sensitive penicillins in pneumonia, the high use of clindamycin for treatment of infections in skin, soft tissue, bone and joint and the unexplained difference in the doses and dosing frequency of cefotaxime and ampicillin between the hospitals.

\section{Author affiliations}

${ }^{1}$ Department of Pediatrics, Møre and Romsdal Hospital Trust, Ålesund, Norway

${ }^{2}$ Faculty of Medicine, Department of Pharmacology, University of Oslo, Oslo, Norway ${ }^{3}$ Department of Drug Statistics, Norwegian Institute of Public Health, Oslo, Norway

${ }^{4}$ Pediatric Department, Oslo University Hospital, Oslo, Norway

${ }^{5}$ Department of Research and Innovation, Møre and Romsdal Hospital Trust, Ålesund, Norway

${ }^{6}$ Department of Infectious Diseases, University of Oslo, Oslo, Norway

Correction notice This article has been corrected since it first published. Supplementary file 2 and Table 3 have been updated.

Contributors CMT, DB, HSB and BHE were involved in the development of the protocol. CMT developed the registration form and was responsible for the data collection at Ålesund Hospital. IA was responsible for the data collection at Oslo University Hospital, Ullevål. CMT and TÅM did the analyses. CMT wrote the first draft. All the authors contributed to the interpretation of the data and revisions of the manuscript and approved the final version of the manuscript.

Funding This work was supported by a grant from Møre and Romsdal Hospital Trust (2018/1527-3).

Competing interests None declared.

Patient consent for publication Obtained.

Ethics approval The study was approved by the Regional Committee for Medical and Health Research Ethics (2017/30/REK Midt) and by the Local Data Protection Officials at the two hospitals.

Provenance and peer review Not commissioned; externally peer reviewed.

Data sharing statement Datafiles with deidentified patient data from the registration in both hospitals are kept by the first author and are available from him upon request. The project protocol is attached in this submission.

Open access This is an open access article distributed in accordance with the Creative Commons Attribution Non Commercial (CC BY-NC 4.0) license, which permits others to distribute, remix, adapt, build upon this work non-commercially, and license their derivative works on different terms, provided the original work is properly cited, appropriate credit is given, any changes made indicated, and the use is non-commercial. See: http://creativecommons.org/licenses/by-nc/4.0/. 


\section{REFERENCES}

1. Roca I, Akova M, Baquero F, et al. The global threat of antimicrobial resistance: science for intervention. New Microbes New Infect 2015;6:22-9.

2. Goossens $\mathrm{H}$. Antibiotic consumption and link to resistance. Clin Microbiol Infect 2009;15(Suppl3):12-15.

3. Aiken AM, Allegranzi B, Scott JA, et al. Antibiotic resistance needs global solutions. Lancet Infect Dis 2014;14:550-1.

4. Korpela K, Salonen A, Virta LJ, et al. Intestinal microbiome is related to lifetime antibiotic use in Finnish pre-school children. Nat Commun 2016;7:10410.

5. Bailey LC, Forrest CB, Zhang P, et al. Association of antibiotics in infancy with early childhood obesity. JAMA Pediatr 2014;168:1063-9.

6. Mitre E, Susi A, Kropp LE, et al. Association Between Use of AcidSuppressive Medications and Antibiotics During Infancy and Allergic Diseases in Early Childhood. JAMA Pediatr 2018;172:e180315.

7. NORM/NORM-VET. Usage of Antimicrobial Agents and Occurrence of Antimicrobial Resistance in Norway. Tromsø / Oslo; 2017. ISSN: 1502-2307 (print) / 1890-9965 (electronic).

8. European Centre for Disease Prevention and Control. Surveillance of antimicrobial resistance in Europe 2016. Annual Report of the European Antimicrobial Resistance Surveillance Network (EARS-Net). Stockholm: ECDC, 2017.

9. Norwegian Ministry of Health and Care Services. National Strategy against Antibiotic Resistance 2015-2020. Report number: I-1164 E. Oslo, 2015.

10. Raastad R, Tvete IF, Abrahamsen TG, et al. A worrying trend in weight-adjusted paediatric antibiotic use in a Norwegian tertiary care hospital. Acta Paediatr 2015;104:687-92.

11. Thaulow CM, Berild D, Eriksen BH, et al. Potential for More Rational Use of Antibiotics in Hospitalized Children in a Country with Low Resistance - Data From Eight Point Prevalence Surveys. Pediatr Infect Dis J 2018;38.

12. Versporten A, Bielicki J, Drapier N, et al. The Worldwide Antibiotic Resistance and Prescribing in European Children (ARPEC) point prevalence survey: developing hospital-quality indicators of antibiotic prescribing for children. J Antimicrob Chemother 2016;71:1106-17.

13. Porta A, Hsia Y, Doerholt K, et al. Comparing neonatal and paediatric antibiotic prescribing between hospitals: a new algorithm to help international benchmarking. $J$ Antimicrob Chemother 2012;67:1278-86.

14. Sharma M, Damlin A, Pathak A, et al. Antibiotic Prescribing among Pediatric Inpatients with Potential Infections in Two Private Sector Hospitals in Central India. PLoS One 2015;10:e0142317.

15. Liem TB, Krediet TG, Fleer A, et al. Variation in antibiotic use in neonatal intensive care units in the Netherlands. J Antimicrob Chemother 2010;65:1270-5.

16. Gharbi M, Doerholt K, Vergnano S, et al. Using a simple pointprevalence survey to define appropriate antibiotic prescribing in hospitalised children across the UK. BMJ Open 2016;6:e012675.

17. De Luca M, Donà $D$, Montagnani $C$, et al. Antibiotic Prescriptions and Prophylaxis in Italian Children. Is It Time to Change? Data from the ARPEC Project. PLoS One 2016;11:e0154662.

18. Di Pietro P, Della Casa Alberighi O, Silvestri M, et al. Monitoring adherence to guidelines of antibiotic use in pediatric pneumonia: the MAREA study. Ital J Pediatr 2017;43:113.

19. Ivanovska V, Hek K, Mantel-Teeuwisse AK, et al. Age-Specific Antibiotic Prescribing and Adherence to Guidelines in Pediatric Patients in Primary Care. Pediatr Infect Dis J 2018;37:218-23.
20. Launay E, Levieux K, Levy C, et al. Compliance with the current recommendations for prescribing antibiotics for paediatric community-acquired pneumonia is improving: data from a prospective study in a French network. BMC Pediatr 2016;16:126.

21. Aseeri MA. The impact of a pediatric antibiotic standard dosing table on dosing errors. J Pediatr Pharmacol Ther 2013;18:220-6.

22. Bielicki JA, Barker Cl, Saxena S, et al. Not too little, not too much: problems of selecting oral antibiotic dose for children. BMJ 2015;351:h5447.

23. Zhang L, Li Y, Zeng L, et al. Applying "children defined daily dose" to assess appropriate dose in pediatrics. J Evid Based Med 2012;5:2-5.

24. Ahmed U, Spyridis N, Wong IC, et al. Dosing of oral penicillins in children: is big child=half an adult, small child=half a big child, baby=half a small child still the best we can do? BMJ 2011;343:d7803.

25. Colijn C, Cohen T. How competition governs whether moderate or aggressive treatment minimizes antibiotic resistance. Elife 2015;4:e10559.

26. Epstein BJ, Gums JG, Drlica K. The changing face of antibiotic prescribing: the mutant selection window. Ann Pharmacother 2004;38:1675-82.

27. European Centre for Disease Prevention and Control. Point prevalence survey of healthcare associated infections and antimicrobial use in European acute care hospitals - protocol version 4.3. Stockholm: ECDC, 2012.

28. Norwegian Pediatric Association. Akuttveileder i Pediatri (in Norwegian). 2013. http://www.helsebiblioteket.no/retningslinjer/ akuttveileder-i-pediatri/forside?hideme=true (Accessed $23 \mathrm{Nov}$ 2017).

29. British Medical Association. BNF for children 2016-2017. 1st ed. London: BMJ Group, Pharmaceutical Press and RCPCH Publications Ltd.

30. McMullan BJ, Andresen D, Blyth CC, et al. Antibiotic duration and timing of the switch from intravenous to oral route for bacterial infections in children: systematic review and guidelines. Lancet Infect Dis 2016;16:e139-e152.

31. Berg AS, Inchley CS, Aase A, et al. Etiology of Pneumonia in a Pediatric Population with High Pneumococcal Vaccine Coverage: A Prospective Study. Pediatr Infect Dis J 2016;35:e69-e75.

32. Hazir T, Nisar YB, Abbasi S, et al. Comparison of oral amoxicillin with placebo for the treatment of world health organization-defined nonsevere pneumonia in children aged 2-59 months: a multicenter, double-blind, randomized, placebo-controlled trial in pakistan. Clin Infect Dis 2011;52:293-300.

33. Felleskatalogen AS. Felleskatalogen (in Norwegian). https://www. felleskatalogen.no/medisin/ (Accessed Mar 2018).

34. Eliakim-Raz N, Robenshtok E, Shefet D, et al. Empiric antibiotic coverage of atypical pathogens for community-acquired pneumonia in hospitalized adults. Cochrane Database Syst Rev 2012;9:CD004418.

35. Brown KA, Khanafer N, Daneman N, et al. Meta-analysis of antibiotics and the risk of community-associated Clostridium difficile infection. Antimicrob Agents Chemother 2013;57:2326-32.

36. Park MA, Li JT, Jt L. Diagnosis and management of penicillin allergy. Mayo Clin Proc 2005;80:405-10.

37. Ben-Shoshan M. Most children labeled as penicillin allergic are at low risk for true penicillin allergy. J Pediatr 2017;188:308-11. 